\title{
Some Estimates of Certain Subnormal and Hyponormal Derivations
}

\author{
Vasile Lauric \\ Department of Mathematics, Florida A\&M University, Tallahassee, FL 32307, USA \\ Correspondence should be addressed to Vasile Lauric, vasile.lauric@famu.edu
}

Received 23 October 2007; Accepted 9 January 2008

Recommended by Nils Ackermann

\begin{abstract}
We prove that if $A$ and $B^{*}$ are subnormal operators and $X$ is a bounded linear operator such that $A X-X B$ is a Hilbert-Schmidt operator, then $f(A) X-X f(B)$ is also a Hilbert-Schmidt operator and $\|f(A) X-X f(B)\|_{2} \leq L\|A X-X B\|_{2}$ for $f$ belongs to a certain class of functions. Furthermore, we investigate the similar problem in the case that $S, T$ are hyponormal operators and $X \in \mathcal{L}(\mathscr{\ell})$ is such that $S X-X T$ belongs to a norm ideal $\left(J,\|\cdot\|_{J}\right)$, and we prove that $f(S) X-X f(T) \in J$ and $\|f(S) X-X f(T)\|_{J} \leq C\|S X-X T\|_{J}$ for $f$ being in a certain class of functions.
\end{abstract}

Copyright (C) 2008 Vasile Lauric. This is an open access article distributed under the Creative Commons Attribution License, which permits unrestricted use, distribution, and reproduction in any medium, provided the original work is properly cited.

\section{Introduction}

Let $\mathscr{\ell}$ be a separable, infinite dimensional, complex Hilbert space, and denote by $\mathcal{L}(\mathscr{\ell})$ the algebra of all bounded linear operators on $\mathscr{H}$ and by $\mathcal{C}_{2}(\mathscr{\ell})$ the Hilbert-Schmidt class. For $T \in \mathcal{L}(\mathscr{L}), \sigma(T)$ denotes the spectrum of $T$, and for a compact subset $\Sigma \subset \mathbb{C}, \operatorname{Lip}(\Sigma)$ denotes the set of Lipschitz functions on $\Sigma$. Furthermore, $\operatorname{Rat}(\Sigma)$ denotes the algebra of rational functions with poles of $\Sigma$, and $R(\Sigma)$ denotes the closure of $\operatorname{Rat}(\Sigma)$ in the supremum norm over $\Sigma$.

For operators $A, B \in \mathcal{L}(\mathscr{\ell})$, the mapping $\Delta_{A, B}(X)=A X-X B$ is called a (generalized) derivation. If $A, B$ are normal (subnormal or co-subnormal, hyponormal or co-hyponormal) operators, then $\Delta_{A, B}$ will be called a normal (subnormal, hyponormal) derivation, respectively.

Next, we recall some theorems that involve normal derivations, and then we extend some of these theorems to the case in which $A, B^{*}$ are subnormal operators and to the case in which $A=S, B=T$ are hyponormal operators.

In [1], a generalization of Fuglede-Putnam theorem for normal operators was proved. For further results concerning normal derivations, the reader can see $[2,3]$.

Theorem 1.1 (see [1]). If $A, B \in \mathcal{L}(\mathscr{L})$ are normal operators and $X \in \mathcal{L}(\mathscr{\ell})$ satisfies $A X-X B \in$ $\mathcal{C}_{2}(\mathscr{L})$, then $A^{*} X-X B^{*} \in \mathcal{C}_{2}(\mathscr{L})$ and 


$$
\|A X-X B\|_{2}=\left\|A^{*} X-X B^{*}\right\|_{2} .
$$

In [4], Furuta extended the above result to subnormal operators.

Theorem 1.2 (see [4]). If $A, B^{*} \in \mathcal{L}(\mathscr{L})$ are subnormal operators and $X \in \mathcal{L}(\mathscr{L})$ satisfies $A X-X B \in$ $\mathcal{C}_{2}(\mathscr{L})$, then $A^{*} X-X B^{*} \in \mathcal{C}_{2}(\mathscr{\ell})$ and

$$
\|A X-X B\|_{2} \geq\left\|A^{*} X-X B^{*}\right\|_{2} .
$$

In his paper [5], Kittaneh proved the following theorem using a famous result of Voiculescu [6] according to which every normal operator can be written as the sum of a diagonal operator and a Hilbert-Schmidt operator of an arbitrarily small Hilbert-Schmidt norm.

Theorem 1.3 (see [5]). Let $A, B \in \mathcal{L}(\mathscr{L})$ be normal operators and $X \in \mathcal{L}(\mathscr{L})$ such that $A X-X B \in$ $\mathcal{C}_{2}(\mathscr{L})$, and let $f \in \operatorname{Lip}(\sigma(A) \cup \sigma(B))$. Then $f(A) X-X f(B)$ is also a Hilbert-Schmidt operator and

$$
\|f(A) X-X f(B)\|_{2} \leq L\|A X-X B\|_{2}
$$

where $L$ is the Lipschitz constant of the function $f$.

\section{Subnormal derivations}

In this section, we investigate the validity of this inequality in the case that $A, B^{*}$ are subnormal operators, but with a drawback concerning the extent of the class of functions in which $f$ can run.

The following lemma is elementary and can be easily established making use of the minimal normal extension of a subnormal operator. Its proof is left for the reader.

Lemma 2.1. If $S_{1}, S_{2} \in \mathcal{L}(\mathscr{d})$ are subnormal operators, then there exists a Hilbert space $\mathcal{K} \supset \mathcal{d e}$ and normal operators $N_{1}, N_{2} \in \mathcal{L}(\mathcal{K})$ that are extensions of $S_{1}, S_{2}$, respectively, and $\sigma\left(N_{i}\right) \subseteq \sigma\left(S_{i}\right), i=$ 1,2

For a subnormal operator $S \in \mathcal{L}(\mathscr{\ell})$ and a function $f \in R(\sigma(S))$, one can associate an operator $f(S) \in \mathcal{L}(\mathscr{L})$ as follows. Let $r_{n} \in \operatorname{Rat}(\sigma(S)), n \in \mathbb{N}$, such that

$$
\left\|f-r_{n}\right\|_{\sigma(S), \infty} \longrightarrow 0, \quad \text { as } n \longrightarrow \infty,
$$

and let $N_{S} \in \mathcal{L}(\mathcal{K})$, where $\mathcal{K} \supset \mathscr{\mathcal { L }}$, be the minimal normal extension of $S$. Since $\sigma\left(N_{S}\right) \subseteq \sigma(S)$, we have

$$
r_{n}\left(N_{S}\right)=\left(\begin{array}{cc}
r_{n}(S) & S_{12}^{\prime} \\
0 & S_{22}^{\prime}
\end{array}\right),
$$

and $r_{n}\left(N_{S}\right) \rightarrow f\left(N_{S}\right)$ in the operator norm of $\mathcal{L}(\mathcal{K})$. Therefore, $r_{n}(S)$ converges in the operator norm of $\mathcal{L}(\mathscr{L})$ to an operator that will be denoted by $f(S)$. It is obvious that this operator does not depend on the sequence $\left\{r_{n}\right\}$. In a similar way, for $f \in R(\sigma(T))$, one can define $f(T)$, when $T^{*} \in \mathcal{L}(\mathscr{\ell})$ is a subnormal operator. 
Theorem 2.2. Let $A, B^{*} \in \mathcal{L}(\mathscr{L})$ be subnormal operators and $X \in \mathcal{L}(\mathscr{L})$ such that $A X-X B \in$ $\mathcal{C}_{2}(\mathscr{L})$, and let $\Sigma=\sigma(A) \cup \sigma(B)$ and $f \in \operatorname{Lip}(\Sigma) \cap R(\Sigma)$. Then $f(A) X-X f(B)$ is also a HilbertSchmidt operator and

$$
\|f(A) X-X f(B)\|_{2} \leq L\|A X-X B\|_{2}
$$

where $L$ is the Lipschitz constant of the function $f$.

Proof. For subnormal operators $A, B^{*} \in \mathcal{L}(\mathscr{\ell})$, according to Lemma 2.1, there exists a Hilbert space $\mathcal{K} \supset \mathscr{H}$ and there are some normal operators $N_{A}, N_{B^{*}} \in \mathcal{L}(\mathcal{K})$ such that relative to the decomposition of $\mathcal{K}=\mathscr{\ell} \oplus \mathscr{\ell}^{\perp}$, we have

$$
N_{A}=\left(\begin{array}{cc}
A & A_{12} \\
0 & A_{22}
\end{array}\right), \quad N_{B^{*}}=\left(\begin{array}{cc}
B^{*} & B_{12} \\
0 & B_{22}
\end{array}\right),
$$

and $\sigma\left(N_{A}\right) \subseteq \sigma(A), \sigma\left(N_{B^{*}}\right) \subseteq \sigma\left(B^{*}\right)$.

If we put $\tilde{X}=X \oplus 0$ on $\mathscr{L} \oplus \mathscr{L}^{\perp}$, then we have $N_{A} \tilde{X}-\tilde{X} N_{B^{*}}^{*}=(A X-X B) \oplus 0$, and therefore $N_{A} \tilde{X}-\tilde{X} N_{B^{*}}^{*} \in \mathcal{C}_{2}(\mathcal{K})$.

For $r \in \operatorname{Rat}(\Sigma)$, where $\Sigma=\sigma(A) \cup \sigma(B)$, a simple calculation shows that

$$
r\left(N_{A}\right)=\left(\begin{array}{cc}
r(A) & A_{12}^{\prime} \\
0 & A_{22}^{\prime}
\end{array}\right), \quad r\left(N_{B^{*}}^{*}\right)=\left(\begin{array}{cc}
r(B) & 0 \\
B_{21}^{\prime} & B_{22}^{\prime}
\end{array}\right) .
$$

Thus, if $f \in \operatorname{Lip}(\Sigma) \cap R(\Sigma)$, using a limiting argument, one can see that $f\left(N_{A}\right)$ and $f\left(N_{B^{*}}^{*}\right)$ have similar matrix representation as in (2.5), but with $f$ replacing $r$. According to Theorem 1.3,

$$
\begin{gathered}
f\left(N_{A}\right) \tilde{X}-\tilde{X} f\left(N_{B^{*}}^{*}\right) \in \mathcal{C}_{2}(\mathcal{K}), \\
\left\|f\left(N_{A}\right) \tilde{X}-\tilde{X} f\left(N_{B^{*}}^{*}\right)\right\|_{2} \leq L\left\|N_{A} \tilde{X}-\tilde{X} N_{B^{*}}^{*}\right\|_{2} .
\end{gathered}
$$

Since $f\left(N_{A}\right) \tilde{X}-\tilde{X} f\left(N_{B^{*}}^{*}\right)=(f(A) X-X f(B)) \oplus 0$, the proof is finished.

Corollary 2.3. Let $A, B^{*} \in \mathcal{L}(\mathscr{L})$ be subnormal operators and $X \in \mathcal{L}(\mathscr{l})$ such that $A X-X B \in$ $\mathcal{C}_{2}(\mathscr{L})$, and let $\Sigma=\sigma(A) \cup \sigma(B)$ and $f \in \operatorname{Lip}(\Sigma) \cap R(\Sigma)$. Then

$$
\left\|f(A)^{*} X-X f(B)^{*}\right\|_{2} \leq\|f(A) X-X f(B)\|_{2^{\prime}}
$$

and thus

$$
\left\|f(A)^{*} X-X f(B)^{*}\right\|_{2} \leq L\|A X-X B\|_{2}
$$

where $L$ is the Lipschitz constant of the function $f$.

Proof. The first inequality is a consequence of Theorem 1.2 after observing that $f(A)$ and $f(B)^{*}$ are subnormal operators. The second inequality follows from Theorem 2.2. 


\section{Hyponormal derivations}

In this section, we approach the same problem, but in the case in which $A=S, B=T$ are hyponormal operators and the Hilbert-Schmidt class is replaced with an arbitrary norm ideal.

For a hyponormal operator $T \in \mathcal{L}(\mathscr{L})$, the analytic functional calculus can be extended to a class $A^{\alpha}(\sigma(T))$ of "pseudo-analytic" functions on $\sigma(T)$ that satisfy a certain growth condition at the boundary.

The extension of the analytic functional calculus for a hyponormal operator was introduced by Dyn'kin (cf. [7, 8]) and it also can be found in [9].

We briefly review the definition and the main tools that are necessary. Let $\Sigma$ be a perfect compact set of the complex plane and let $\alpha$ be a positive noninteger with $k$ its integer part, $[\alpha]$. The class $A^{\alpha}(\Sigma)$ is defined as the set of $(k+1)$ tuples of continuous functions on $\Sigma,\left(f_{0}, \ldots, f_{k}\right)$ : $\Sigma \rightarrow \mathbb{C}^{k+1}$ that are related by

$$
\begin{gathered}
f_{j}(z)=f_{j}\left(z_{0}\right)+\frac{f_{j+1}\left(z_{0}\right)}{1 !}\left(z-z_{0}\right)+\cdots+\frac{f_{k}\left(z_{0}\right)}{(k-j) !}\left(z-z_{0}\right)^{k-j}+R_{j}\left(z_{0}, z\right), \\
\left|R_{j}\left(z_{0}, z\right)\right| \leq C_{j}\left|z-z_{0}\right|^{\alpha-j}
\end{gathered}
$$

for $j=0, \ldots, k$ and $z, z_{0} \in \Sigma$. Since $\Sigma$ is a perfect set,

$$
f_{j}\left(z_{0}\right)=\lim _{z \rightarrow z_{0}} \frac{f_{j-1}(z)-f_{j-1}\left(z_{0}\right)}{z-z_{0}}, \quad j=0, \ldots, k-1,
$$

and thus the $(k+1)$ tuple depends only on $f_{0}$. The space $A^{\alpha}(\Sigma)$, endowed with the maximum of the smallest constants that satisfy (3.1) plus the supremum norm on $\Sigma$ of $f_{0}$, becomes a unital Banach algebra and is a closed subalgebra of $\operatorname{Lip}(\alpha, \Sigma)$, the algebra of Lipschitz functions of order $\alpha$.

Theorem 3.1 (see [8]). Let $\Sigma$ be a perfect compact set, $f \in C(\Sigma)$, and $\alpha$ a positive noninteger. The following are equivalent:

(a) $f \in A^{\alpha}(\Sigma)$;

(b) $f$ has an extension $F \in C^{1}(\mathbb{C} \backslash \Sigma)$ with $|\bar{\partial} F(z)| \leq C \cdot \operatorname{dist}(z, \Sigma)^{\alpha-1}, z \notin \Sigma$;

(c) there exists $\phi \in C_{0}(\mathbb{C})$ such that

$$
f(z)=\int \frac{\phi(w)}{w-z} d \mu(w), \quad z \in \Sigma,
$$

and $|\phi(w)| \leq C_{0} \cdot\|f\|_{A^{\alpha}(\Sigma)} \cdot \operatorname{dist}(w, \Sigma)^{\alpha-1}, w \in \mathbb{C}$, where $\mu$ is planar Lebesgue measure and $C_{0}$ is a constant that does not depend on $f$.

If $T \in \mathcal{L}(\mathscr{\ell})$ is a hyponormal operator, then $\|T\|=\|T\|_{\sigma}$, where $\|T\|_{\sigma}$ denotes the spectral radius of $T$, that is $\sup _{z \in \sigma(T)}|z|$. It is well known that if $z \notin \sigma(T)$, then $(z-T)^{-1}$ is also hyponormal and thus

$$
\left\|(z-T)^{-1}\right\|=\frac{1}{\operatorname{dist}(z, \sigma(T))}
$$


Thus, for a hyponormal operator $T$ whose spectrum $\sigma(T)$ is a perfect set and for a function $f \in A^{\alpha}(\sigma(T))$ with $\alpha>2$, one can associate an operator defined by

$$
\int \phi(w)(w-T)^{-1} d \mu(w)
$$

that will be denoted by $f(T)$. The above integral does not depend on $\phi$, that is, the definition of $f(T)$ is not ambiguous, and the mapping $\phi \mapsto f(T)$ acting from $A^{\alpha}(\sigma(T))$ into $\mathcal{L}(\mathscr{\ell})$ is a continuous, unital morphism of Banach algebras, and which extends the Riesz-Dunford calculus.

Let $\left(J,\|\cdot\|_{J}\right)$ be a norm ideal, that is, a proper two-sided ideal $J$ of $\mathcal{L}(\mathscr{L})$ with a norm $\|\cdot\|_{J}$ that satisfies th following: $\left(J,\|\cdot\|_{J}\right)$ is a Banach space and $\|A X B\|_{J} \leq\|A\|\|B\|\|X\|_{J}$, for all $X \in J$ and any $A, B \in \mathcal{L}(\mathscr{\ell})$. In particular, the Shatten-von Neumann $p$-classes, $\mathcal{C}_{p}(\mathscr{\ell})$, for $p \geq 1$, are instances of norm ideals.

Theorem 3.2. Let $\left(J,\|\cdot\|_{J}\right)$ be a norm ideal, let $S, T \in \mathcal{L}(\mathscr{L})$ be hyponormal operators for which both $\sigma(S)$ and $\sigma(T)$ are perfect sets, let $f$ belong to $A^{\alpha}(\Sigma)$ with $\alpha>3$ and $\Sigma=\sigma(S) \cup \sigma(T)$, and let $X \in \mathcal{L}(\mathscr{L})$ such that $S X-X T \in J$. Then $f(S) X-X f(T) \in J$ and

$$
\|f(S) X-X f(T)\|_{J} \leq C_{1} \cdot\|f\|_{A^{\alpha}(\Sigma)} \cdot\|S X-X T\|_{J^{\prime}}
$$

where $C_{1}$ is a constant that depends on $\Sigma$ but it does not depend on $f$.

Proof. For $f \in A^{\alpha}(\Sigma)$, according to Theorem 3.1, there exists $\phi \in C_{0}(\mathbb{C})$ such that

$$
\begin{gathered}
f(z)=\int \frac{\phi(w)}{w-z} d \mu(w), \quad z \in \Sigma, \\
|\phi(w)| \leq C_{0} \cdot\|f\|_{A^{\alpha}(\Sigma)} \cdot \operatorname{dist}(w, \Sigma)^{\alpha-1}, \quad w \in \mathbb{C} .
\end{gathered}
$$

Therefore,

$$
f(S) X-X f(T)=\int \phi(w)\left[(w-S)^{-1} X-X(w-T)^{-1}\right] d \mu(w) .
$$

The domain of integration is $\operatorname{supp}(\phi)$, which is a compact set that has in common with $\Sigma$ only possibly boundary points of $\Sigma$. For $w \in \operatorname{supp}(\phi) \cap(\mathbb{C} \backslash \Sigma)$,

$$
\begin{aligned}
(w-S)^{-1} X-X(w-T)^{-1} & =(w-S)^{-1}[X(w-T)-(w-S) X](w-T)^{-1} \\
& =(w-S)^{-1}[S X-X T](w-T)^{-1} \in J,
\end{aligned}
$$

and, according to (3.4),

$$
\begin{aligned}
\left\|(w-S)^{-1} X-X(w-T)^{-1}\right\|_{J} & \leq \operatorname{dist}(w, \sigma(S))^{-1} \cdot \operatorname{dist}(w, \sigma(T))^{-1} \cdot\|S X-X T\|_{J} \\
& \leq C^{\prime} \cdot \operatorname{dist}(w, \Sigma)^{-2} \cdot\|S X-X T\|_{J}
\end{aligned}
$$

where $C^{\prime}$ is a constant that depends on $\Sigma$. Therefore, the integrant in (3.8) belongs to the norm ideal $J$ and

$$
\left\|\phi(w)(w-S)^{-1} X-X(w-T)^{-1}\right\|_{J} \leq C_{0} \cdot C^{\prime} \cdot\|f\|_{A^{\alpha}(\Sigma)} \cdot \operatorname{dist}(w, \Sigma)^{\alpha-3}\|S X-X T\|_{J},
$$


for $w \in \operatorname{supp}(\phi) \cap(\mathbb{C} \backslash \Sigma)$. After integration one obtains the desired conclusion of the theorem.

\section{References}

[1] G. Weiss, "The Fuglede commutativity theorem modulo the Hilbert-Schmidt class and generating functions for matrix operators. II," Journal of Operator Theory, vol. 5, no. 1, pp. 3-16, 1981.

[2] G. Weiss, "The Fuglede commutativity theorem modulo operator ideals," Proceedings of the American Mathematical Society, vol. 83, no. 1, pp. 113-118, 1981.

[3] G. Weiss, "An extension of the Fuglede commutativity theorem modulo the Hilbert-Schmidt class to operators of the form $\Sigma M_{n} X N_{n}$," Transactions of the American Mathematical Society, vol. 278, no. 1, pp. $1-20,1983$.

[4] T. Furuta, "An extension of the Fuglede-Putnam theorem to subnormal operators using a HilbertSchmidt norm inequality," Proceedings of the American Mathematical Society, vol. 81, no. 2, pp. 240-242, 1981.

[5] F. Kittaneh, "On Lipschitz functions of normal operators," Proceedings of the American Mathematical Society, vol. 94, no. 3, pp. 416-418, 1985.

[6] D. Voiculescu, "Some results on norm-ideal perturbations of Hilbert space operators," Journal of Operator Theory, vol. 2, no. 1, pp. 3-37, 1979.

[7] E. M. Dyn'kin, "An operator calculus based on the Cauchy-Green formula. Investigations on linear operators and the theory of functions, III," Zapiski Naučnyh Seminarov Leningradskogo Otdelenija Matematičeskogo Instituta im. V. A. Steklova Akademii Nauk SSSR (LOMI), vol. 30, pp. 33-39, 1972, (Russian).

[8] E. M. Dyn'kin, "Pseudoanalytic continuation of smooth functions. Uniform scale," in Mathematical Programming and Related Questions (Proc. Seventh Winter School, Drogobych, 1974), Theory of Functions and Functional Analysis (Russian), pp. 40-73, Central Èkonom.-Mat. Inst. Akad. Nauk SSSR, Moscow, Russia, 1976.

[9] M. Martin and M. Putinar, Lectures on Hyponormal Operators, vol. 39 of Operator Theory: Advances and Applications, Birkhäuser, Basel, Switzerland, 1989. 


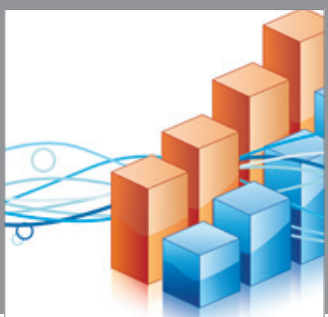

Advances in

Operations Research

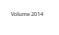

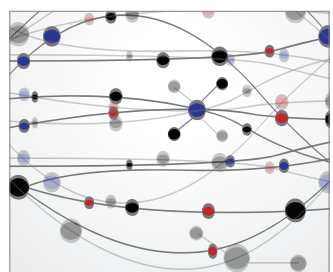

\section{The Scientific} World Journal
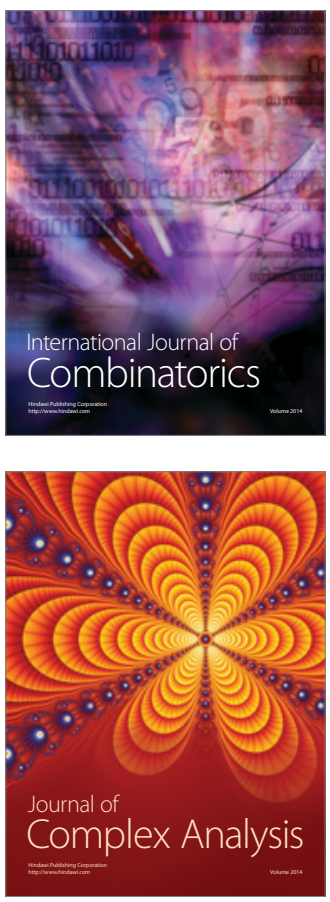

International Journal of

Mathematics and

Mathematical

Sciences
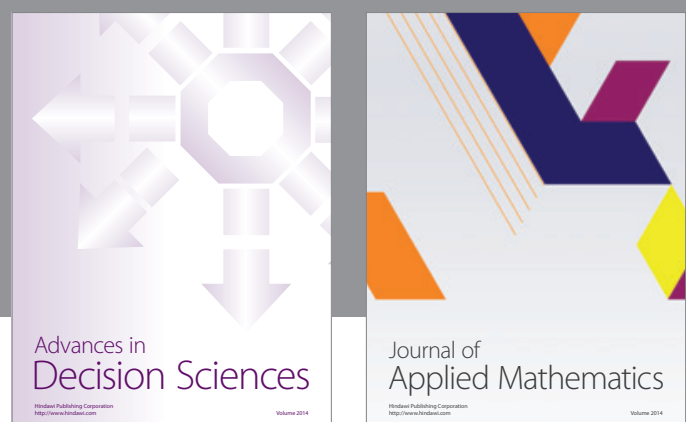

Journal of

Applied Mathematics
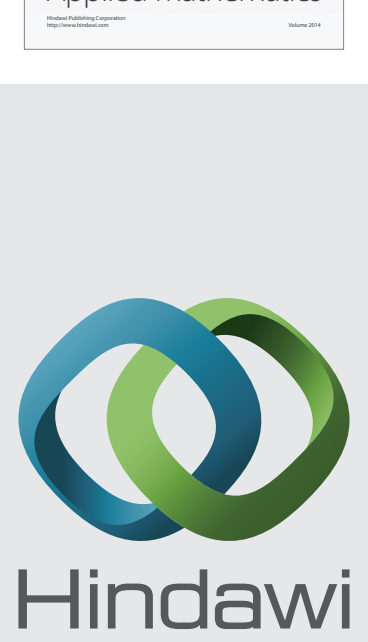

Submit your manuscripts at http://www.hindawi.com
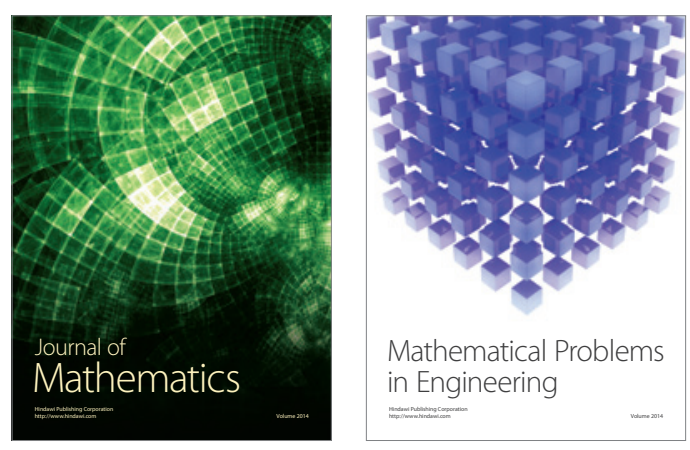

Mathematical Problems in Engineering
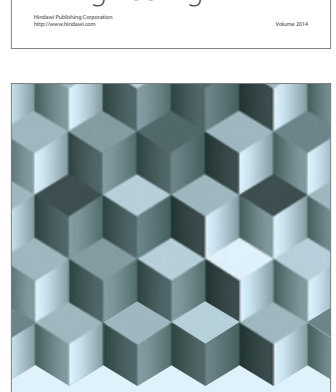

Journal of

Function Spaces
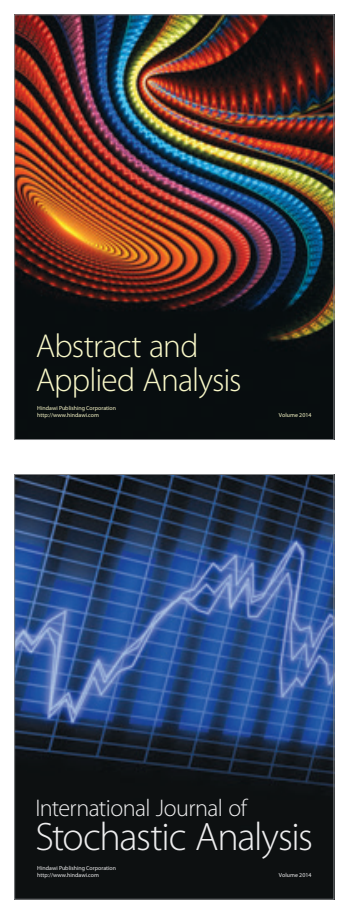

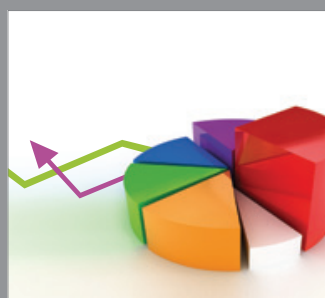

ournal of

Probability and Statistics

Promensencen
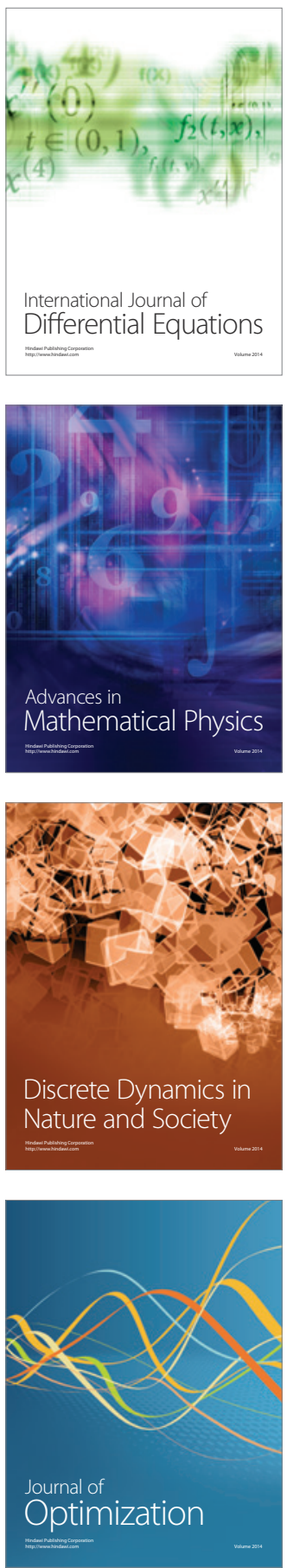